\title{
Factores asociados a la infección respiratoria aguda en niños menores de cinco años. ENDES, 2017
}

\author{
Factors associated with acute respiratory infection in children under five \\ years of age. ENDES, 2017 \\ Miriam Ramírez-Guevara ${ }^{1}$ \\ Ambar Cárdenas-López ${ }^{1}$ \\ Delia Dávila-Chachapoyas ${ }^{1}$
}

\begin{abstract}
Resumen
Objetivo: determinar los factores sociodemográficos asociados a la infección respiratoria aguda en niños peruanos menores de 5 años. Materiales y métodos: estudio descriptivo correlacional de corte transversal a partir de un análisis secundario de la ENDES, 2017. La muestra efectiva del estudio fue de 18345 madres que tenían hijos menores de cinco años. Se tomó en cuenta como variable principal a la infección respiratoria aguda de los niños y a los factores las características sociodemográficas de la madre. Para el análisis descriptivo de las variables categóricas se emplearon frecuencias y porcentajes. En el análisis inferencial se emplearon las pruebas Chi-cuadrado y T de Student. Resultados: el 14.7\% de los niños presentó infección respiratoria aguda. Los factores asociados significativamente a la infección respiratoria aguda fueron quintil de riqueza $(\mathrm{p}=0.001)$ y región $(\mathrm{p}=0.05)$. Conclusión: el estudio muestra que el $14.4 \%$ de los niños presenta infección respiratoria aguda. Asimismo, el quintil de riqueza inferior y vivir en la región selva son factores importantes para la presencia de una infección respiratoria aguda en los menores de cinco años. El person al de salud debe tener en cuenta estos hallazgos en sus labores de promoción y prevención sobre las IRAS, específicamente la neumonía.
\end{abstract}

Palabras clave: Infecciones Neumocócicas; Salud del Niño; Infecciones del Sistema Respiratorio (Fuente: DeCS).

\begin{abstract}
Objective: to determine the sociodemographic factors associated with acute respiratory infection in Peruvian children under 5 years. Materials and methods: the study was a descriptive cross-sectional correlation based on a secondary analysis of ENDES, 2017. The effective sample of the study was 18345 mothers who had children under five years of age. Acute respiratory infection of children was taken into account as the main variable and the sociodemographic characteristics of the mother were factors. For the descriptive analysis of the categorical variables frequencies and percentages were used. In the inferential analysis, Chi-square and Student's T tests were used. Results: $14.4 \%$ of the children presented acute respiratory infection. The factors significantly associated with acute respiratory infection were the wealth quintile $(p=0.001)$ and the region $(p=0.05)$. Conclusion: the study shows that $14.4 \%$ of children have an acute respiratory infection. Likewise, the lowest wealth quintile and living in the jungle region are important factors for the presence of acute respiratory infection in children under five years of age. The health personnel must take into account these findings in their promotion and prevention work on the HAIs, specifically pneumonia.
\end{abstract}

Keys words: Pneumococcal Infections; Child Health; Respiratory Tract Infections (Source: DeCS).

Para citar:

Ramírez M, Cárdenas A, Dávila D. Factores asociados a la infección respiratoria aguda en niños menores de cinco años. CASUS. 2019;4(1):1-7.

${ }^{1}$ Hospital II-1 Rioja. Licenciada de Enfermería.

Correo electrónico: ramirezguevaramiriam18@gmail.com
Fecha de recepción: 28-02-19

Fecha de envío a pares: 03-03-19

Fecha de aprobación por pares : 29-03-19

Fecha de aceptación: 08-04-19 


\section{INTRODUCCIÓN}

La infección respiratoria aguda (IRA) es causada predominantemente por bacterias (Streptococcus pneumoniae y Haemophilus influenzae) y virus, además es una causa importante de morbilidad y mortalidad en niños a nivel mundial afectando la salud pública (1). La IRA incluye a todas las infecciones del tracto respiratorio, siendo la infección respiratoria baja aguda la que en su mayor parte carga con la gravedad de la enfermedad (1). Aproximadamente la IRA causa el $20 \%$ de todas las muertes en niños en edad preescolar en todo el mundo, y el $90 \%$ de estas muertes se debe a la neumonía (1).

Según la Organización Mundial de la Salud (OMS) la neumonía es la causa principal de morbilidad en los países en desarrollo con índice de 0.29 episodios por niño y año, un 0.05 episodios por niño y año en los países desarrollados. La mayoría de los casos se dan en la India (43 millones), China (21 millones), Pakistán (10 millones), entre otros países en desarrollo (2). Asimismo, en el 2015 la neumonía provocó un $15 \%$ de todas las defunciones en niños menores de cinco años (3).

En el 2013 en el Perú se presentaron 29994 casos de neumonías, es decir una tasa de 103.4 por cada 10 mil menores de cinco años (4). En los años 2009-2013 la tasa de incidencia del país estuvo en el rango de 4.8 a 34.4 , siendo el promedio nacional de 11.5 por 1000 menores de cinco años. Las mayores tasas de incidencia se registraron en los departamentos de Ucayali, Loreto, Pasco, Arequipa, Madre de Dios y Huánuco (4). Por otro lado, "la tasa de mortalidad media por neumonía varió entre 2.8 a 48.3 , siendo el promedio país de 1.9" (4). Las tasas mayores corresponden al departamento de la región selva con mayor incidencia y de mayor mortalidad en neumonías. En el 2013, el 49\% de las muertes en menores de cinco años ocurrieron en la sierra, $34 \%$ en la selva y $17 \%$ en la costa (4).

Debido a la alta prevalencia, incidencia y mortalidad de la IRA en niños menores de cinco años a nivel mundial y nacional, es importante reconocer los factores de riesgo que conllevan a adquirir esta infección. La literatura menciona los factores de riesgo los antecedentes fisiopatológicos y biológicos asociados a la IRA en niños (5). Sin embargo, no solo se deberían tomar en cuenta estos factores, sino a los sociodemográficos como el quintil de riqueza. Debido a que, generalmente, las familias que pertenecen a un quintil inferior viven en comunidades rurales con un estilo inadecuado de vida, las madres descuidan la higiene de sus hijos, prevalecen las habitaciones compartidas con adultos y/o otros niños. En estas condiciones los menores son los más susceptibles a contraer infecciones respiratorias agudas, principalmente la neumonía $(6,7)$. Además, se debe tener en cuenta en la región donde vive la madre con su hijo. El clima y las condiciones ambientales varían notablemente entre las regiones del Perú. En este sentido, son numerosas las zonas de la región selva del Perú donde el personal de salud no puede llegar para realizar actividades promocionales y preventivas por motivos de distancia, falta de acceso o por la presencia de desastres naturales frecuentes (8).

Teniendo en cuenta estos factores que afectan la salud de los niños menores de cinco de años es importante que el personal de salud, en especial de enfermería, identifique a las madres que no pueden adquirir o requieren información sobre la salud de sus hijos, porque ellas son las principales cuidadoras (9). Además, las actividades preventivas promocionales sobre enfermedades comunes en niños, como las IRAS, principalmente la neumonía, entre otras no deberían ser limitadas (10). Por tal motivo el objetivo del estudio fue determinar los factores sociodemográficos asociados a la infección respiratoria aguda en niños peruanos menores de 5 años.

\section{MATERIALES Y MÉTODOS}

El estudio fue no experimental, descriptivo y correlacional de corte transversal a partir de un análisis secundario de la base de datos de la Encuesta Demográfica y de Salud familiar (ENDES) del año 2017 direccionada por el Instituto Nacional de Estadística e Informática (INEI) (11). El muestreo fue bietápico 
por conglomerados y estratificados de zonas rurales y urbanas de los departamentos del Perú. Como criterios de selección se incluyeron a madres de 15 a 45 años de edad con hijos menores de 5 años, por lo que la muestra efectiva del estudio fue de 18345 madres. actualmente trabaja, cesárea, fumar como lactancia materna fueron variables cualitativas dicotómicas (sí y no).

La información de las encuestadas fue recolectada por el Instituto Nacional de Estadística e

Figura 1. Prevalencia de la infección respiratoria aguda en niños menores de cinco años, 2017

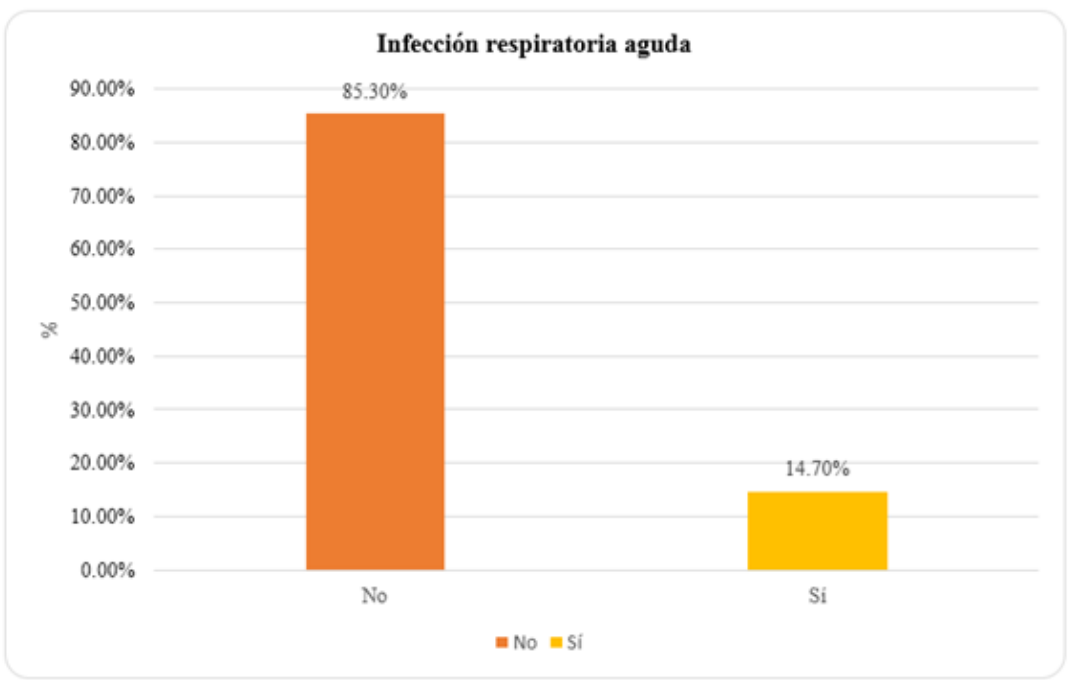

La variable principal fue infección respiratoria aguda (IRA) definida como la presencia de manera conjunta de tos y dificultad para respirar o respiración rápida, en las dos semanas previas a la encuesta. Variable dicotómica (sí y no) medida a partir de las siguientes preguntas: ¿En los últimos 14 días el niño o niña ha tenido tos? y ¿Respiraba más rápido que de costumbre, con respiraciones cortas y agitadas? (12). Estas preguntas fueron respondidas por las madres.

Respecto a los factores sociodemográficos fueron edad de la madre, considerada una variable cuantitativa discreta; quintil de riqueza, definida como la riqueza del hogar respecto a la disponibilidad de bienes y servicios y de las características del hogar, por ejemplo, el acceso a servicios básicos de agua potable y la presencia de servicios higiénicos, entre otros consumos que se da en la vivienda, variable cualitativa politómica (quintil inferior, segundo quintil, quintil intermedio, cuarto quintil y quintil superior) (13); región, variable cualitativa politómica (costa, sierra y selva); nivel educativo, variable cualitativa politómica (sin educación, primaria, secundaria y superior); tipo de residencia, variable cualitativa dicotómica (urbano y rural); tanto las variables
Informática (INEI). Este organismo recolecta datos acerca de la salud de la mujer y sus hijos menores de cinco años en todo el Perú mediante una entrevistadora capacitada (14). Se respetó la confidencialidad de las respuestas para el anonimato de las entrevistadas y se brindó un consentimiento informado verbal. Respecto al cuestionario individual se tomaron en cuenta las preguntas y respuestas de los siguientes módulos o secciones: antecedentes de la entrevista; embarazo, parto, puerperio y lactancia y trabajo de la mujer (15).

Para el análisis de los datos se utilizó el programa estadístico STATA versión 14. Se calcularon frecuencias y porcentajes para las variables cualitativas y para las cuantitativas las medidas de tendencia central. Además, se aplicó la prueba estadística de Chi-cuadrado para la relación entre dos variables cualitativas. Por otro lado, se utilizó la prueba de Shapiro Wilk para determinar la normalidad de las variables cuantitativas. Una vez determinados los presupuestos se utilizó la prueba de $\mathrm{T}$ de Student. Se consideró un nivel de significancia estadística menor o igual a 0.05 . 


\section{RESULTADOS}

En la tabla 1 se muestra que del total de las madres la media de la edad fue de 30.3 años y la media de número de hijos fue 2.4. Asimismo al momento de la encuesta, el $42.2 \%$ de todas las madres con sus hijos vivió en la región costa, el $72.4 \%$ residió en una zona urbana y el $27.3 \%$ perteneció a un segundo quintil de riqueza, el $58.0 \%$ trabajaba y el $46.8 \%$ contó con educación secundaria. Por último, el $63.4 \%$ brindó lactancia materna inmediata, el $98.1 \%$ no resultó fumador y el $68.0 \%$ no se realizó una cesárea.

En la figura 1 se observa que el $85.2 \%$ de los niños menores de cinco años no presentó infección respiratoria aguda y el $14.7 \%$ presentó esta infección.

En la tabla 2 se evidencia la relación significativa entre la edad de las madres y la infección respiratoria aguda en sus hijos $(\mathrm{p}=0.001)$. Sin embargo, las medias en años de edad fueron similares tanto para las madres que tienen tuvieron hijos con infección respiratoria aguda (media=29.0) como para las que no tuvieron hijos con esta patología (media=30.1). Asimismo, se encontró la relación significativa entre el quintil de riqueza de las madres y la infección respiratoria aguda en los niños ( $\mathrm{p}=0.001)$. En donde el 16.3\% de las madres que pertenecen al quintil inferior tiene hijos que presentan IRA. Por otro lado, se observó relación significativa entre la región en que vivían las madres e hijos y las infecciones respiratorias agudas en sus menores $(\mathrm{p}=0.05)$. Es decir, en los últimos 14 días a la encuesta el 15.3\% de los niños que vivieron en la región selva presentaron IRA.

\section{DISCUSIÓN}

En el estudio solo el $14.7 \%$ de los niños menores de cinco años presentó una infección respiratoria aguda. Se determinó la asociación significativa entre los factores sociodemográficos (quintil de riqueza y región) y la infección respiratoria aguda en niños peruanos menores de cinco años. Las madres que pertenecen a un quintil de riqueza inferior y viven en la región selva del Perú tienen hijos que presentaron IRA.
Tabla 1. Características de las madres de los niños menores de 5 años

\begin{tabular}{|c|c|c|}
\hline & $\mathbf{n}$ & $\%$ \\
\hline \multicolumn{3}{|c|}{ Lactancia materna inmediata } \\
\hline No & 6672 & 36.5 \\
\hline Sí & 11597 & 63.5 \\
\hline Edad $(\mathbf{m} \pm \mathbf{D S})$ & 30.3 & 9.2 \\
\hline \multicolumn{3}{|l|}{ Actualmente trabaja } \\
\hline No & 7700 & 41.9 \\
\hline Sí & 10645 & 58.1 \\
\hline Número de hijos $(m \pm D S)$ & 1.9 & 1.7 \\
\hline \multicolumn{3}{|l|}{ Cesárea } \\
\hline No & 12482 & 68.1 \\
\hline Sí & 5863 & 31.9 \\
\hline \multicolumn{3}{|l|}{ Fumar } \\
\hline No & 17996 & 98.1 \\
\hline Sí & 349 & 1.9 \\
\hline \multicolumn{3}{|l|}{ Nivel educativo } \\
\hline Sin educación & 287 & 1.6 \\
\hline Primaria & 3582 & 19.5 \\
\hline Secundaria & 8590 & 46.8 \\
\hline Superior & 5886 & 32.1 \\
\hline \multicolumn{3}{|l|}{ Quintil de riqueza } \\
\hline Quintil inferior & 4705 & 25.6 \\
\hline Segundo quintil & 5025 & 27.3 \\
\hline Quintil intermedio & 3887 & 21.3 \\
\hline Cuarto quintil & 2849 & 15.6 \\
\hline Quintil superior & 1879 & 10.2 \\
\hline \multicolumn{3}{|l|}{ Tipo de residencia } \\
\hline Urbano & 13289 & 72.5 \\
\hline Rural & 5056 & 27.5 \\
\hline \multicolumn{3}{|l|}{ Región } \\
\hline Costa & 7749 & 42.4 \\
\hline Sierra & 6766 & 36.8 \\
\hline Selva & 3830 & 20.8 \\
\hline
\end{tabular}

Un hallazgo del estudio fue que las mujeres que pertenecen al quintil de riqueza inferior tienen hijos que presentan infección respiratoria aguda. En un estudio se reporta asociación entre las infecciones respiratorias agudas y tener un bajo nivel económico (5). Este hallazgo se explicaría, por ejemplo porque los miembros de la familia con niveles socioeconómicos bajos, generalmente, viven hacinados en la vivienda donde en una sola habitación duermen más de tres personas. Si un miembro presenta una enfermedad respiratoria asintomática, por el contacto directo con los demás, contribuye al contagio especialmente de los niños y adultos mayores (16). Además, no contar con recursos económicos se relaciona con el mal estilo de vida de las familias y niveles inferiores de educación, donde las madres brindan alimentos inadecuados para sus hijos e incluso no brindan 
Tabla 2. Asociación descriptiva entre los factores y la infección respiratoria aguda en niños menores de cinco años

\begin{tabular}{|c|c|c|c|}
\hline & \multicolumn{3}{|c|}{ Infección respiratoria aguda } \\
\hline & $\begin{array}{c}\text { No } \\
\text { n }(\%)\end{array}$ & $\begin{array}{c}\text { Sí } \\
\text { n(\%) }\end{array}$ & p \\
\hline $\begin{array}{l}\text { Lactancia materna } \\
\text { inmediata }\end{array}$ & & & 0.51 \\
\hline No & $5703(85.5)$ & $969(14.5)$ & \\
\hline Sí & 9872 (85.2) & 1725 (14.8) & \\
\hline Edad* & $30.1 \pm 7.0$ & $29.0 \pm 6.9$ & 0.001 \\
\hline Actualmente trabaja & & & 0.61 \\
\hline No & $6553(41.9)$ & $9088(58.10)$ & \\
\hline Sí & $1147(42.42)$ & $1557(57.58)$ & \\
\hline Número de hijos* & $2.40 \pm 1.54$ & $2.35 \pm 1.58$ & 0.13 \\
\hline Cesárea & & & 0,19 \\
\hline No & 10613 & 1869 (14.9) & \\
\hline Sí & $5028(85.7)$ & $835(14.3)$ & \\
\hline Fumar & & & 0.14 \\
\hline No & $15353(85.4)$ & $2643(14.6)$ & \\
\hline Sí & $288(82.6)$ & $61(17.4)$ & \\
\hline Educación & & & 0.14 \\
\hline Sin educación & $244(85.1)$ & $43(14.9)$ & \\
\hline Primaria & $3016(84.2)$ & $566(15.8)$ & \\
\hline Secundaria & $7322(85.3)$ & $1268(14.7)$ & \\
\hline Superior & $5059(85.9)$ & $827(14.1)$ & \\
\hline Quintil de riqueza & & & 0.001 \\
\hline Quintil inferior & $3935(83.6)$ & $770(16.4)$ & \\
\hline Segundo quintil & $4265(84.8)$ & $760(15.2)$ & \\
\hline Quintil intermedio & $3335(85.8)$ & $552(14.2)$ & \\
\hline Cuarto quintil & $2453(86.1)$ & $396(13.9)$ & \\
\hline Quintil superior & $1653(87.9)$ & $226(12.1)$ & \\
\hline Lugar de residencia & & & 0.24 \\
\hline Urbano & $11355(85.4)$ & 1934 (14.6) & \\
\hline Rural & $4286(84.77)$ & $770(15.23)$ & \\
\hline Región & & & 0.05 \\
\hline Costa & $6578(84.8)$ & $1171(15.2)$ & \\
\hline Sierra & $5821(86.1)$ & $945(13.9)$ & \\
\hline Selva & $3242(84.6)$ & $588(15.4)$ & \\
\hline
\end{tabular}

lactancia materna exclusiva y por último no vacunan a sus menores contra la neumonía (9). En consecuencia los niños presentan más predisposición a adquirir una infección respiratoria aguda entre otras enfermedades (17).

Otro hallazgo importante del estudio fue la relación significativa entre la región y la infección respiratoria aguda, en donde las madres que vivían en la Selva tenían hijos con infección respiratoria aguda. Otros estudios sobre el tema no reportan una relación entre ambas variables como indica el presente estudio. Sin embargo, una parte de la explicación de este hallazgo tiene relación con la alimentación durante los seis primeros meses de vida el bebé donde debe recibir lactancia materna exclusiva (LME). Es un fenómeno frecuente que las madres en esta Región interrumpan esta alimentación aportando otro tipo de alimento (18). La literatura reporta que si una madre brinda LME a su bebé previene la neumonía (18). Por otro lado, la región Selva está compuesta por zonas húmedas y lluviosas que favorecen una mayor exposición y vulnerabilidad ante los agentes que causan una infección respiratoria aguda $(7,19)$. Además, hay extensas áreas de la Región donde existen limitaciones en la accesibilidad de las madres a los puestos y/o centros de salud por la distancia. O viceversa, respecto al personal de salud en realizar visitas domiciliarias impedido por los frecuentes desastres naturales como por ejemplo lluvias e inundaciones (20). Estas dificultades limitan que el personal de salud realice sus actividades preventivas promocionales en cuanto a las IRAS y otras enfermedades.

Una de las limitaciones del estudio está vinculada a la calidad de las respuestas de las madres durante la entrevista debido a sesgos de memoria. Por otro lado, respecto a la prevalencia de IRA esta no fue determinada por un diagnóstico médico sino por la identificación de signos y síntomas por las mismas madres. Asimismo, pese a que la ENDES brinda varios factores asociados a la IRA, aún se requiere de otros referentes a la alimentación, antecedentes patológicos personales o familiares del menor, acceso a un centro de salud, etc. Por esta razón se recomienda tener en cuenta los factores mencionados en futuros estudios. No obstante las limitaciones señaladas se debe tomar en cuenta como fortaleza del estudio la muestra representativa a nivel nacional.

\section{CONCLUSIONES}

En conclusión, el estudio muestra que al momento de la encuesta $14.7 \%$ de los niños peruanos presentó síntomas de infección respiratoria aguda. Asimismo, el quintil de riqueza inferior y vivir en la región selva son factores importantes para la presencia de una infección respiratoria aguda en el menor. 
A partir de estos hallazgos el personal de salud debe tomar en cuenta las características sociodemográficas de las madres y de su hogar en sus labores de promoción y prevención sobre las IRAS, específicamente la neumonía. Muchas veces las actividades del personal de salud se limitan por problemas de accesibilidad porque los pobladores de zonas alejadas no puedan llegar a un centro de salud o el personal de salud a sus hogares. Teniendo en cuenta que el conocimiento de las madres que conducen a una identificación temprana de la enfermedad probablemente evita una enfermedad grave. Para ello, es necesario un trabajo en conjunto con la población y entidades nacionales para resolver este problema.

\section{REFERENCIAS BIBLIOGRÁFICAS}

1. Bezerra PG, Britto MC, Correia JB, Maria do Carmo MB, Fonceca $\mathrm{AM}$, Rose $\mathrm{K}$, et al. Viral and atypical bacterial detection in acute respiratory infection in 7 . children under five years. PloS one. 2011;6(4):e18928.

2. Organización Mundial de la Salud. Epidemiología y etiología de la neumonía en la niñez [internet]. 8 Ginebra, Suiza: OMS; 2008 [citado el 28 de enero del 2019]. Disponible en: https://www.who.int/bulletin/volu mes/86/5/07-048769-ab/es/

3. Organización Mundial de la Salud. Neumonía [internet].Ginebra, Suiza: OMS; 2016 [citado el 039 Marzo, 2018]. Disponible en: https://www.who.int/es/ne ws-room/fact-

sheets/detail/pneumonia

4. Organización Panamericana de la Salud. Infecciones Respiratorias Agudas en el Perú [internet]. Washington D. C, Estados Unidos: OPS; 2014 [ citado el 28 de enero del 2019]. Disponible en: http://iris.paho.org/xmlui/bitstream /handle/123456789/28549/iras 201 4-spa.pdf

5. Bayona Y, Niederbacher J. Infecciones respiratorias virales en pediatría: generalidades sobre fisiopatogenia, diagnóstico y algunos desenlaces clínicos. Revista Médicas UIS. 2015;28(1):2-3.

6. Aguirre E, Céspedes E. Morbilidad por infecciones respiratorias agudas en niños menores de 5 años. MEDISAN 2014;18(11):4-7.

Rodríguez A, González I. Factores de riesgos asociados a las infecciones respiratorias agudas en lactantes, Veguitas 2013.Multimed. 2014;18(1):70-75.

Servicio Nacional de Meteorología e Hidrología. Clima/Mapa climático del Perú [internet]. Lima, Perú: SENAMHI; 2019 [citado el 28 de enero del 2019]. Disponible en: https://www.senamhi.gob.pe/?p=m apa-climatico-del-peru

Ortiz N. Ortiz K. Características del menor y de la madre asociadas a la neumonía en niños menores de cinco años. CASUS. 2018;3(3):135-138.

10. Gómez M, Zapata R Conocimiento, práctica del 15. Instituto Nacional de Estadística e cuidador y factor pronóstico de infecciones respiratorias agudas en niños.

2017;17(2):125.

11. Instituto Nacional de Estadística e Informática. De la Encuesta Demográfica de la salud Familiar [internet]. Lima, Perú: INEI; 2017 [citado el 30 de enero 2019]. Disponible en: https://webinei.inei.gob.pe/anda_i nei/index.php/catalog/306/vargrp/ VG21

12. Rojas C, Ysla M, Riega V, Ramos O, Moreno C, Bernui I. Enfermedades diarreicas, infecciones respiratorias $y$ características de la alimentación de los niños de 12 a 35 meses de edad en el Perú. Revista peruana de medicina experimental y salud pública. 2004;21(3): 146-156.

13. Enríquez Y, Ortiz K, Ortiz Y. Análisis de los determinantes próximos e impacto de la ocupación en la fertilidad de mujeres peruanas. Revista Panamericana de Salud Pública. 2017;41(2); e18. Disponible en: https://www.scielosp.org/pdf/rpsp/ 2017.v41/e18/es

14. Instituto Nacional de Estadística e Informática. Manual de la entrevista 2016 [internet]. Lima, Perú: INEI; 2016 [citado el 30 de enero de 2019]. Disponible en: http://iinei.inei.gob.pe/iinei/sriena ho/Descarga/Documentos Metodol ogicos/2016-

5/ManualEntrevistadora.pdf

Informática. Cuestionario
individual de la Encuesta Demográfica de salud Familiar Lima, Perú: INEI; 2016 [citado el 30 de enero del 2019]. Disponible en:

https://proyectos.inei.gob.pe/endes /recursos/CUESTIONARIOINDI VIDUAL2016.pdf

16. Rodríguez LCM, Gutiérrez GD, Rodríguez HA, Lastres IH, Mendieta RF. Algunos factores de riesgo de infecciones respiratorias agudas en menores de 5 años. Mayo 2014-2015. Multimed. 2017;21(2):4-13 
17. Gómez P, Consuelo C. Madres adolescentes, un reto frente a los factores que influyen en la lactancia materna. Enfermería 19. Aliaga E, Serpa K. Factores de global. 2014;33(13):63-65.

18. Paredes M. Factores de Riesgo para Infecciones Respiratorias Agudas en Niños Menores de 1 año. C.S. Santa Fe - Callao. 2014.
Revista peruana de obstetricia y 20. Gestión. Lluvias, huaicos, enfermería. $2015 \quad 11(1)$

inundaciones y heladas: el 'pan nuestro' de todos los años en Perú [internet]. Lima, Perú: Gestión; 2017 [citado el 31 de enero del 2019]. Disponible en: https://gestion.pe/economia/lluvias -huaicos-inundaciones-heladaspan-nuestro-anos-peru-129010 